\title{
The unequal efficacy of vinegar in first aid protocols for Pelagia noctiluca (Cnidaria: Scyphozoa) and Carybdea marsupialis (Cnidaria: Cubozoa) stings.
}

\author{
Ainara Ballesteros ${ }^{1 *}$, Macarena Marambio ${ }^{1}$, Veronica Fuentes ${ }^{1}$, Mridvika Narda ${ }^{2}$, Andreu Santín ${ }^{1}$ and Josep- \\ Maria Gili ${ }^{1}$ \\ 1 ICM-CSIC - Institute of Marine Sciences, Department of Marine Biology and Oceanography, Passeig \\ Marítim de la Barceloneta 37-49, 08003 Barcelona, Spain; gili@icm.csic.es (J.M.G.) \\ 2 ISDIN, Innovation and Development, C. Provençals 33, 08019 Barcelona, Spain; mridvika.narda@gmail.com \\ (M.N.) \\ * Correspondence: ballesteros@icm.csic.es (A.B.)
}

\begin{abstract}
Although the jellyfish species that inhabit the Mediterranean coastal waters are not lethal, their sting can cause severe pain and systemic effects that pose a health risk to humans. Despite the frequent occurrence of jellyfish stings, currently no consensus exists regarding the most appropriate first aid protocol among the scientific community. Over the years, several different rinse solutions have been proposed of which vinegar, or acetic acid, is one of the most noticeable as a rinse solution with efficacy data published. We investigated the effect of vinegar and seawater on the nematocyst discharge process in species representative of the Mediterranean region such as Pelagia noctiluca (Scyphozoa) and Carybdea marsupialis (Cubozoa) by means of: (1) direct observation on the nematocyst discharge by light microscope (Tentacle solution assay) and (2) the quantification of hemolytic area (Tentacle skin blood agarose assay). The nematocyst discharge of both species was not stimulated by seawater, and it was classified as a neutral solution. In $P$. noctiluca, vinegar produced nematocyst discharge per ser while inhibiting the nematocyst discharge of $C$. marsupilais. These results suggest that the use of vinegar cannot be universally recommended. While in case of a cubozoan $C$. marsupialis sting, the inhibitory effect of vinegar makes it the best rinse solution par excellence, in case of a scyphozoan $P$. noctiluca sting, its application can be counterproductive, worsening the pain and discomfort of the stung area.
\end{abstract}

Keywords: jellyfish; sting; first aid; vinegar; seawater; nematocyst discharge; Pelagia noctiluca; Carybdea marsupualis.

\section{Introduction}

The Cnidaria phylum is a diverse group with more than 16,000 species distributed non-homogeneously over the world including jellyfish [1,2]. The distintive cells responsible for the cnidarian stings are called cnidocysts [3]. Nematocysts are capsules placed inside the cnidocyts where the toxins are stored along with a coiled tubule. The cnidocyst has a cover located at the top, named operculum, which opens when the suitable chemical or mechanical stimuli are detected by the cnidocil. The activation process causes the nematocyst discharge where the venom is expelled [4].

Jellyfish blooms are increasing in size and frequency in some areas worldwide in recent years [5,6]. Human activities, such as overfishing, eutrophication, translocation climate change and habitat modification, are suggested as the causes of the increase of jellyfish outbreaks [6]. One of the main problems of jellyfish blooms in coastal areas, that include beaches, is the impact on public health and tourism [7-10].

The increase of jellyfish bloom in some areas of the Mediterranean Sea, specifically near the Catalan coast (Spain) in the northwestern region of the Mediterranean, has already been reported [11]. Spain is the country with the second largest number of foreign visitors per year [12]. Most are attracted by the sun and sand model of the tourism industry, making beaches one of the region's major assets [13]. However, beaches can 
become unsafe when risk of exposure to jellyfish, with its known effect on human health [7-10], is high. This is especially true in the summer season, when an encounter between jellyfish and humans is more likely. 150 million jellyfish stings are reported worldwide annually [14], being one of the most requested first aid assistance made to lifeguards during bathing seasons. In Spain, around $60 \%$ of the total assists made by lifeguards at beaches correspond to jellyfish stings [15]. In the region of Catalonia, this number is up to $80 \%$ in some beaches [16].

The Catalan coast is dominated mainly by species from the Scyphozoa class like Pelagia noctiluca, Rhizostoma pulmo and Cotylorhiza tuberculata [17]. Due to its wide distribution, abundance and the severity of its sting, P. noctiluca has become the most important jellyfish of the Mediterranean [18,19]. As such it is responsible for a high number of stings assists carried out by the rescue services in Catalan beaches [11]. $P$. noctiluca has been classified as a high stinging species due to the severity of its sting that generally causes immediate pain, urticaria, edema, burning sensation, formation of vesicles, papules and/or scabs [18, 20, 21].

Carybdea marsupialis is the only representative of the Cubozoa class in some areas of the Mediterranean basin [17,22]. Since the summer of 2008, the presence of this box jellyfish in some areas of the Spanish coast, like Denia (Alicante), has been a significant health problem with thousands of related sting assists required during the summer months [22]. Belonging to the most toxic class of cnidarians, C. marsupialis is not considered a lethal species, but its stings may produce painful skin lesions like vesicles, red papules and/or burning. Systemic symptoms like heart and/or neurological complications are also reported [21, 23, 24].

The first aid protocols against jellyfish stings define a set of actions that allow immediate assistance to persons stung by jellyfish. Current protocols include the removal of jellyfish fragments attached to the skin after the sting followed by, washing the affected area with a rinse solution $[10,17,21,25,26]$. The chemical or mechanical stimulation of remaining undischarged cnidocysts in the skin can lead to a new venom load inoculation. In the sting management scenario, avoiding the second envenomation episode by any means is essential. For this reason, most first aid protocols against jellyfish stings are focused mainly on rinsing of the affected area with a solution, which helps remove the residual cnidocysts without their activation [10, 17, 21, 25, 26].

As of today, there is no consensus on the most appropriate rinse solution to wash the affected area producing a wide debate among the scientific community. Recommendations range from seawater, vinegar, urine and alcohol with vinegar or acetic acid, being the most studied and the one that generates the greatest debate [10, 21, 25, 26] While some research groups support the universal use of vinegar [27] regardless of species type, others suggest the reevaluation of the currently recommended rinse solutions [10] and more research to discover new compounds [25]. However, these recommendations must take into account the differences in nematocyst morphologies, nematocyst types or the different response in front of the same stimuli between them. Considering this, prior to their recommendation as a post-sting rinse solution, these ought to be subjected to species-specific tests in order to determine their efficacy against a particular species, avoiding the extrapolation of results that may end in bad practices of the first aid protocols.

The objective of the present study was to determine: (I) the effect of vinegar and seawater rinse solutions in the nematocyst discharge process using Tentacle solution assay (TSA) methodology and (II) the inhibitory effect of vinegar in the nematocyst discharge (TSA) and the venom load by means of Tentacle skin blood agarose assay (TSBAA) method in P. noctiluca, the dominant venomous species, and C. marsupialis, the only jellyfish of the Cubozoa class present in the Mediterranean coast.

\section{Results}

2.1 Evaluation of nematocyst discharge (TSA) 
Pelagia noctiluca. Nematocysts were not activated after the incubation on seawater (Figure 1A and Table 1). As such, seawater was classified as a neutral solution (Table 1). Conversely, nematocyst discharge was observed immediately after the incubation of the tentacle with vinegar (Figure 1B-D). All nematocyst types were activated (Figure 1B,C). The vinegar rinse solution was classified as activator solution (Table 1).

Carybdea marsupilais. Nematocyst discharge was not recognized after seawater and vinegar incubation (Figure 2A, C). In this first phase, both rinse solutions were classified as neutral solutions (Table $\mathbf{1}$ ).
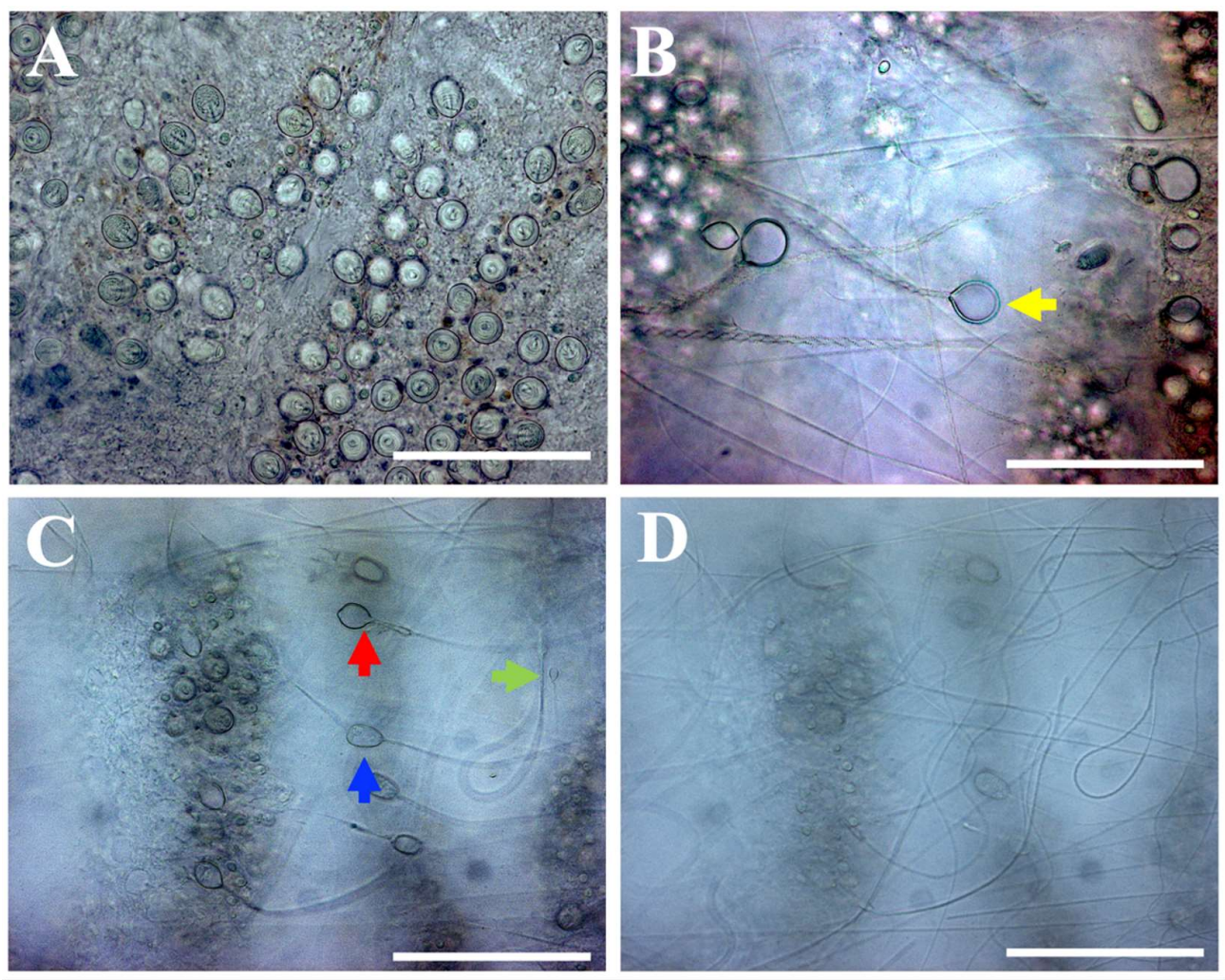

Figure 1. Nematocyst response of P.noctiluca after the incubation on: (A) seawater, (B-D) vinegar. Note in the presence of vinegar: (B) discharged O-isorhiza (yellow arrow), (C) discharged a-isorhiza (green arrow), A-isorhiza (blue arrow) and eurytele (red arrow), (D) all tubules discharged. Scale bars: $100 \mu \mathrm{m}$.

Table 1. Nematocyst response effect of $P$. noctiluca and C. marsupialis after the application of seawater (control) and vinegar and $\mathrm{pH}$ values.

\section{P. noctiluca C. marsupialis}

\begin{tabular}{llllllll}
\hline $\begin{array}{l}\text { Rinse } \\
\text { solution }\end{array}$ & $\mathbf{p H}$ & $\mathbf{n}$ & Discharge $^{1}$ & Effect $^{2}$ & $\mathbf{n}$ & Discharge $^{1}$ & Effect $^{2}$ \\
\hline Seawater & 7.80 & 6 & 0 & Neutral & 3 & 0 & Neutral \\
Vinegar & 2.60 & 6 & ++ & Activator & 3 & 0 & Neutral \\
\hline
\end{tabular}

${ }^{1}$ Nematocyst discharged categories: 0 = no discharge was observed; ++ = medium discharge of nematocysts.

${ }^{2}$ Rinse solution effect categories: activator solution effect = nematocysts activated after the incubation on the solution; neutral solution effect = nematocysts not activated after the incubation on the solution.

$n$ indicates the number of replicates. 

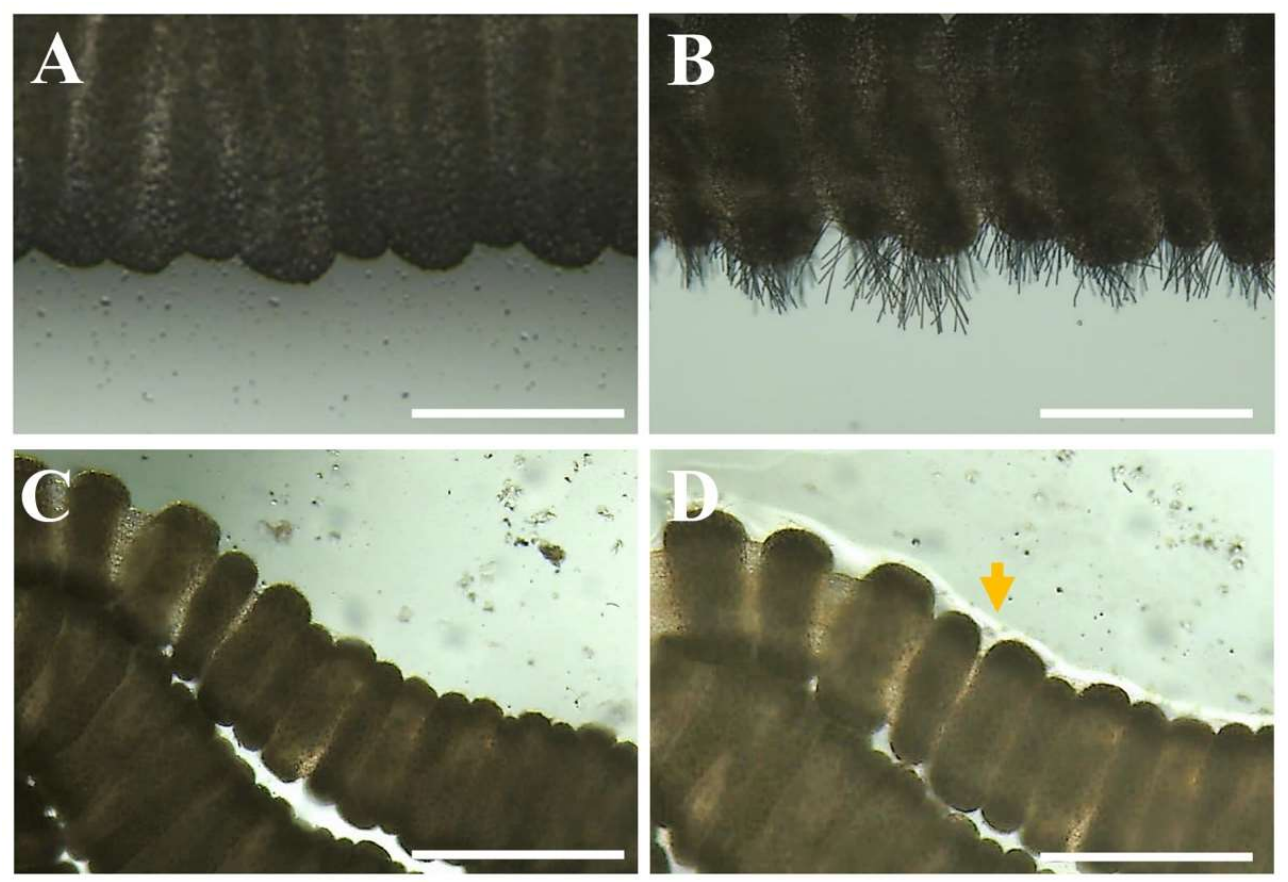

Figure 2. Nematocyst response of C. marsupialis after the incubation on: (A) seawater, (B) ethanol, (C) vinegar. Note the different response between a neutral solution (A) and an activator solution per se (B). (D) Inhibitory effect after the first incubation on vinegar and the subsequent application of ethanol. Note the pass of ethanol through the tentacle (orange arrow). Scale bars: $(A, B) 1 \mathrm{~mm},(C, D)$ $0.5 \mathrm{~mm}$.

\subsection{Evaluation of the inhibitory effect (TSA and TSBAA)}

To determine the inhibitory effect of the rinse solutions (TSA), only neutral solutions were considered. However, the the venom load experiments (TSBAA) were performed for both seawater and vinegar.

Pelagia noctiluca. Chemical nematocyst stimulation with TSA was not performed due to the activation observed with vinegar activation per se (Figure 1B-D) (section 2.1). Hemolytic areas observed after the sting in the presence of seawater and vinegar are presented in Figures 3A, B, 4 and Table 3. Both hemolytic area values were similar indicating that neither solution tested inhibited nematocyst discharge in this species (Table 3).

Carybdea marsupilais. After the first incubation on the two rinse solutions (seawater and vinegar), the tentacles were activated by chemical stimulation by means of ethanol as activator solution (Figure 2B). Preincubation of tentacles with seawater lead to the discharge of nematocysts when activated by ethanol (Table 2). The hemolysis area observed after the sting process after seawater incubation is presented in Figures 3C, 4 and Table 3). Seawater was classified as a neutral solution (Table 2).

Vinegar was classified as an inhibitory solution, where it was able to completely inhibit the nematocyst discharge even when exposed to an ethanol solution (Figure 2D, orange arrow). The hemolytic area data also reflected its inhibitory effect (Figures 3D, 4 and Table 3). Vinegar acted as an inhibitory solution, preventing the nematocyst discharge and reducing, almost entirely, hemolysis (Figure 4 and Tables 2, 3).

Table 2. Inhibitory efficacy of seawater and vinegar on the nematocyst discharge in C. marsupialis. 


\begin{tabular}{llll}
\hline Rinse solution & $\mathbf{n}$ & Discharge $^{1}$ & Effect $^{2}$ \\
\hline Ethanol & 3 & +++ & Activator \\
Seawater + ethanol & 3 & +++ & Neutral \\
Vinegar + ethanol & 3 & 0 & Inhibitory \\
\hline${ }^{1}$ Nematocyst discharged categories: $0=$ no discharge was observed; $+++=$ maximum discharge of \\
nematocysts. \\
2Rinse solution effect categories: activator solution effect $=$ nematocysts activated after the \\
incubation on the solution; inhibitory solution effect= nematocysts not activated after the incubation \\
in a first solution but neither by the consecutive stimulation by a known activator (ethanol). \\
$n$ indicates the number of replicates.
\end{tabular}
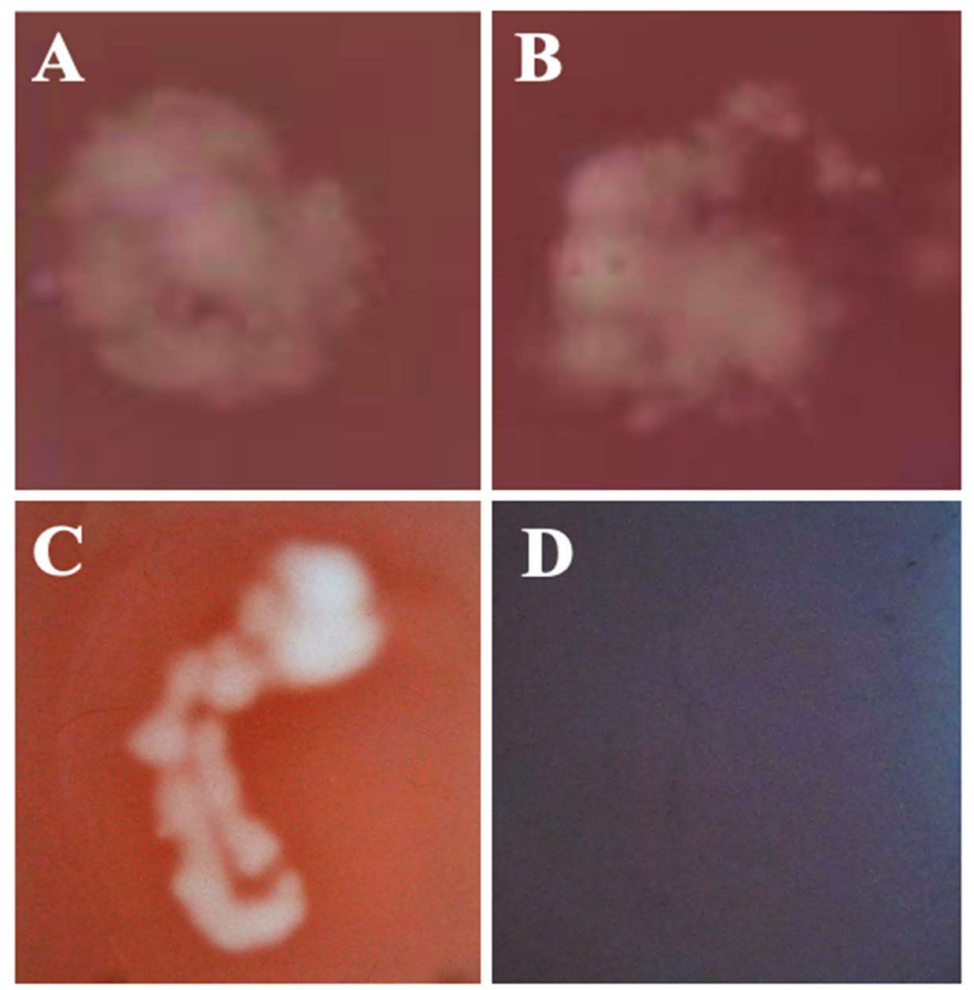

Figure 3. Hemolytic areas produced by the venom of (A-B) P. noctiluca, after the contact with: (A) seawater, (B) vinegar and (C-D) C. marsupialis, after the contact with: (C) seawater, (D) vinegar.

Table 3. Inhibitory efficacy of vinegar on the nematocyst discharge in P. noctiluca and C. marsupialis.

\begin{tabular}{ccccccc}
\hline & \multicolumn{3}{c}{ Pelagia noctiluca } & \multicolumn{3}{c}{ Carybdea marsupialis } \\
\hline $\begin{array}{c}\text { Rinse } \\
\text { solution }\end{array}$ & $\mathbf{n}$ & $\begin{array}{c}\text { Hemolytic } \\
\text { area } \mathbf{( \% )}\end{array}$ & Effect & n & $\begin{array}{c}\text { Hemolytic } \\
\text { area (\%) }\end{array}$ & Effect \\
\hline Seawater & 5 & $23.80 \pm 6.90$ & No inhibitory & 10 & $32.49 \pm 19.33$ & No inhibitory \\
Vinegar & 5 & $21.64 \pm 11.98^{\text {ns }}$ & No inhibitory & 10 & $0.73 \pm 2.31^{* * *}$ & Inhibitory \\
\hline
\end{tabular}

The inhibitory effect of vinegar was tested using a Mann-Whitney test for non-parametric data, results being expressed as follows: ns: $p>0.05$ (not significant), ${ }^{*} p=<0.05,{ }^{* *} p=<0.01,{ }^{* * *} p=<0.001$. $n$ indicates the number of replicates. 


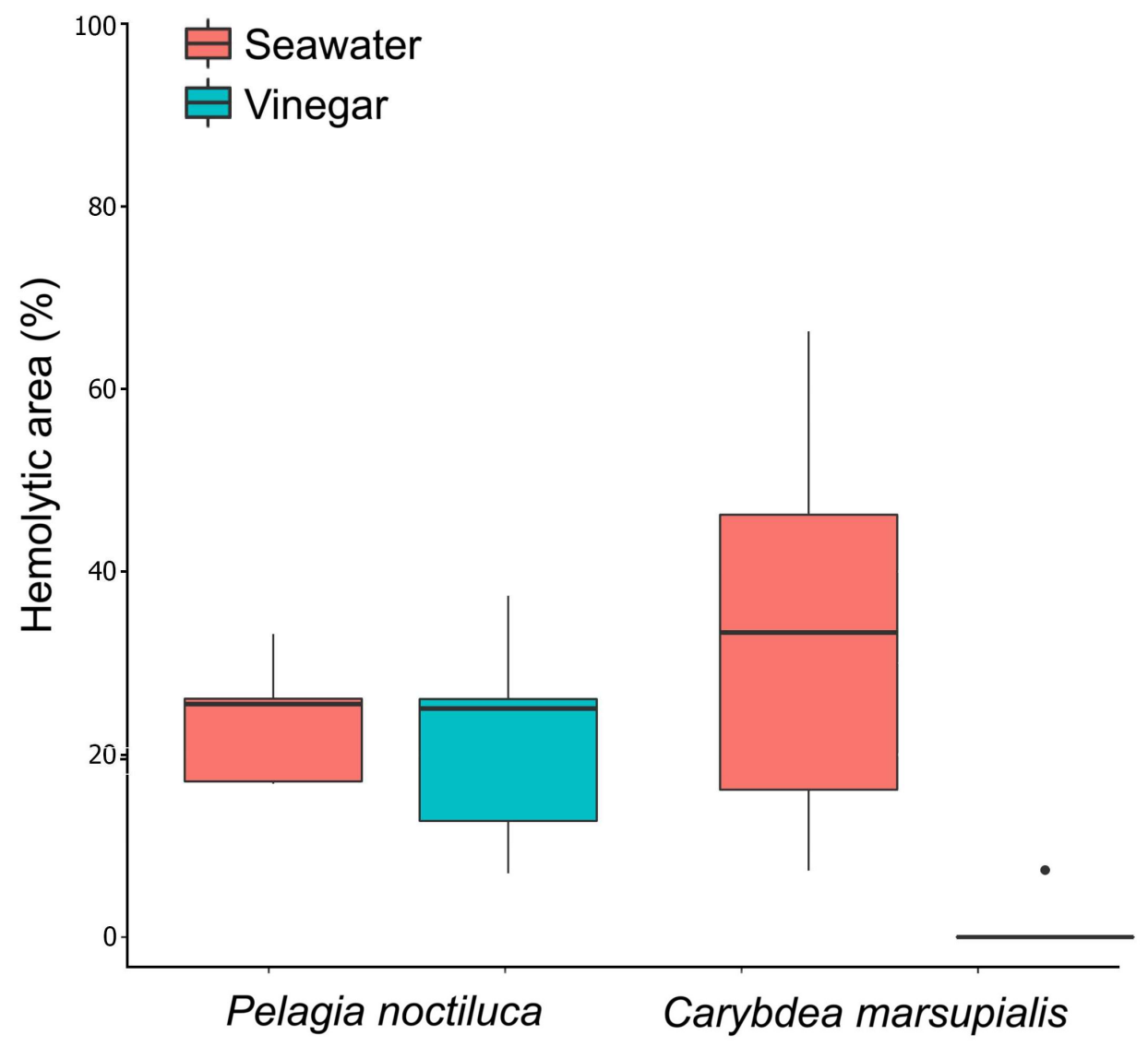

Figure 4. Hemolytic area (\%) produced by de venom load of $P$. noctiluca and C. marsupialis venoms. Note the inhibitory effect of vinegar in the case of C. marsupialis.

\section{Discussion}

Due to the enormous health and socio-economic impact of jellyfish stings in coastal areas, identifying solutions to effectively inhibit the nematocyst discharge is of great importance as these can then be employed to rinse affected area post-sting. Publications suggest the use of vinegar as an ideal rinse solution to safely remove residual jellyfish tissue, without risk of further activation of remaining cnidocysts, irrespective between some classes of the Cnidaria phylum (Cubozoa, Hydrozoa and Scyphozoa) [27]. However, some previous studies have shown that the response of the nematocyst discharge among different jellyfish species or even classes may not be similar [28, 29].

In the present study, we compared the effect of vinegar on the nematocyst discharge in two representative jellyfish of different classes; P. noctiluca (Scyphozoa class) and C. marsupialis (Cubozoa class), both found in the Mediterranean Sea adjoining the Spanish coast $[11,17,22]$. In the current study, vinegar was identified as an inhibitor of discharge for $C$. marsupialis as it was capable of successfully inhibiting the discharge even when the nematocyst discharge was induced by an activator solution (Figure 2D and Table 1). On the contrary, it was identified as an activator per se for the nematocysts of the P. noctiluca jellyfish (Figures 1B-D).

Previous researches did not show nematocyst discharge after the addition of vinegar or acetic acid for cubozoan jellyfish, generating an inhibitory effect or a significant reduction in the venom load as reported in our study for C. marsupialis [28-33]. However, the nematocyst discharge and increased exacerbation of pain intensity are well-known in presence of vinegar or acid acetic for the Scyphozoa class [28, 29,34]. Contrary to our results, Morabito et al. (2014) [35] concluded the effectiveness of 5\% acetic acid as inhibitory solution for $P$. noctiluca recomending it as a local treatment after its sting. 
However, our work shows that the application of vinegar caused immediate nematocyst discharge and hemolytic area due to the penetrating venom into the RBC's agarose (Figures 1B-D, 3B, 4, 5). The differences in the results between both studies can be attributed to the methodology used. In Morabito et al. (2014) [35], the nematocyst discharge was induced by a combined physico-chemical stimulation by chemo-sensitizers in the presence of $5 \%$ acetic acid, followed by mechanical stimulation by a non-vibrating test probe. In contrast, we used a TSA methodology, a technique widely used all over the world [28-30,32,33] and easy to implement. The observation of the rinse solution effect directly on the discharge of nematocysts avoids possible methodological errors. Moreover, in accordance with our findings (Table 1), nematocyst discharge for $P$. noctiluca had been reported previously for extreme acidic $\mathrm{pH}$ values [36]. Since our TSA results were later corroborated by the TSBAA methodology (Figures 3B,4), we conclusively state that the use of vinegar or acetic acid after a $P$. noctiluca sting can lead to further discharge from nematocysts and is clearly detrimental.

A recent study with the scyphozoan Cyanea capillata [27] concluded that vinegar caused a decrease, almost complete, in the venom activity by TSBAA methodology despite its direct nematocyst discharge stimulation by TSA method. The authors suggested that these contrary results could be attributed to the non-discharge of Aisorhiza and $\mathrm{O}$-isorhiza nematocysts, being the types that most contribute to the hemolytic activity. Activation of A-isorhiza and O-isorhiza in the presence of acetic acid was reported formerly for scyphozoan species, including C. capillata [29, 37]. Our study support the discharge of A-isorhiza and O-isorhiza types for scyphozoan jellyfish (Figures 1B, C).

The presence of a physical barrier (pig intestine) in the TSBAA methodology was the modification proposed by Yanagihara et al. (2016) [32] to avoid the solution-induced hemolysis or effect-masking color change. The pig intestine was pretreated by lanolin anhydrous in order to prevent leaks, especially in the case of vinegar. Pretreating the pig intestine skin with a lanolin layer can reduce leaks, but in turn, it can act as a physical barrier preventing the penetration of the nematocyst's tubules into the SRBC's agarose. From our experience, we recommend working without the presence of lanolin in TSBAA experiments whenever possible and in case its application is necessary, a thin layer should be used to ensure that the reduction of the hemolytic area is only due to the effect of the solution on the venom load.

Seawater did not stimulate the discharge process in scyphozoa and cubozoa species $[27-29,32,33]$. Its neutral effects have been confirmed for P. noctiluca and C. marsupialis (Figures 1A, 2A, 3A, C, 4). Because of its neutral condition, some authors suggested that seawater is not a good recommendation to wash the sting area, as nematocysts eventually roll off skin and the discharge may occur due to mechanical effect [27, 32, 33]. Nevertheless, seawater reduced pain and redness in cubozoa and scyphozoa species [28, 29]. Due to its unclear efficacy, it is difficult to conclude the implications of the seawater in first aid protocols.

All the evidence described above reflect the variability of the response of nematocyst discharge in presence of vinegar. The activation of $P$. noctiluca nematocysts prevent the universal use of vinegar and opens new fields of research to explore new rinse solutions that may be used universally in all species. Such a solution is possible if its efficacy can be demonstrated in different classes or species, but if not, species-specific first aid protocols must be established.

\section{Conclusion}

Jellyfish stings account for a large percentage of total assists on beaches. To minimize the adverse effects from jellyfish stings, it is important to establish the guidelines of the first aid protocols based on scientific evidence and to improve them in order to create species-specific protocols. 
Our research has shown that the response of the nematocysts discharge process is different between classes of the phylum Cnidaria. Different species of jellyfish respond differently to the vinegar rinse solution. This species specificity must be kept in mind when prescribing first aid protocols to attend jellyfish stings. Vinegar has demonstrated efficacy for inhibiting the nematocyst discharge in cubozoan species. However, our research has shown that vinegar, or commercial products based on this compound, cannot be recommended in case of stings related to $P$. noctiluca, the predominant venomous species found on the Spanish Mediterranean coast. Its use can be counterproductive causing a direct discharge of the nematocysts, which can worsen the pain of the affected person.

To conclude, we recommend the use of vinegar as an inhibitory rinse solution for $C$. marsupialis. On the contrary, for treating a $P$. noctiluca sting, the use of seawater is suggested due to their neutral effect until a solution with an inhibitory effect can be identified.

\section{Materials and Methods}

\subsection{Collection and preparation of jellyfish}

P. noctiluca individuals were collected in ocean waters in adjoining Barcelona (Catalonia, Spain) during November 2018 and C. marsupialis individuals were collected along the Denia coasts (Alicante, Spain) during September 2018. Both samplings were performed during night. In case of cubozoans, a light was used in order to attract them. Individuals were collected using hand nets and plastic jars to avoid any tissue damage. The specimens were kept in plastic bags with seawater avoiding air bubbles inside, in order to keep the jellyfish in the best conditions during the transport. All experiments were conducted in experimental aquaria zone of the Marine Science Institute (ICM-CSIC) in Barcelona (P. noctiluca) and Montgó Research Station in Denia (C. marsupialis). The day after sampling, the tentacles of both species were cut using dissection scissors and stored in recipients with micro filtered seawater at $4^{\circ} \mathrm{C}$ until their use (less than 48 hours).

\subsection{Rinse solutions and $p H$ measurements}

The rinse solution were seawater (control) and vinegar (Vivó, 6\% acetic acid). The $\mathrm{pH}$ value of each solution was measured with a benchtop $\mathrm{pH}$ meter (Orion Star A211).

\subsection{Evaluation of nematocyst discharge (TSA)}

The methodology used was Tentacle solution assay (TSA) [32]. Tentacles pieces of approximately $3 \mathrm{~cm}$ long were transferred to slides $(76 \times 26 \mathrm{~cm})$. The samples were observed under the light microscope in order to ensure their integrity. Subsequently, tentacles were incubated on microwells with $3 \mathrm{ml}$ of seawater and vinegar during 5 minutes. The preparation was covered carefully with the coverslip and placed under a light microscope in order to observe the nematocyst response.

The nematocyst response was classified qualitatively in four categories in accordance with Pyo et al. (2016) [28]:

1. 0 : no discharge was observed;

2. +: low discharge of nematocysts;

3. ++: medium discharge of nematocysts;

4. +++: high discharge of nematocysts.

The effect of the rinse solution was classified in one of two categories:

1. Activator effect solution: nematocysts were activated after the incubation on the solution;

2. Neutral effect solution: nematocysts were not activated after the incubation on the solution. 


\subsection{Evaluation of the inhibitory effect}

\subsubsection{Nematocyst discharge (TSA)}

In order to know the inhibitory effect in $C$. marsupialis, the nematocyst discharge was chemically stimulated by means of ethanol (PANREAC, ethanol 96\%).

After the seawater and vinegar incubations (section 5.3), $15 \mu \mathrm{l}$ of ethanol were applied on the tentacle.

The effect was classified in one of two categories:

1. Neutral effect solution: nematocysts were not activated after the incubation in a first solution but yes by the consecutive chemical stimulation of ethanol solution;

2. Inhibitory effect solution: nematocysts were not activated after the incubation in a first solution but neither by the consecutive chemical stimulation of ethanol solution.

\subsubsection{Venom load (TSBAA)}

The venom load following contact with the tentacle was evaluated by the quantification of the hemolytic area in a modified protocol adapted from the Tentacle skin blood agarose assay (TSBAA) method published by Yanagihara et al. (2016) [32]. Briefly, an agarose gel preparation incorporating sheep red blood cells (SRBC's) (Thermo Fisher Scientific) was used covered by thin tissue of pig intestine to simulate the effect of the human skin barrier.

A solution of $4 \%$ low melting point agarose (INVITROGEN TM LMP Agarose) in phosphate buffered saline (PBS) (SIGMA) was prepared and cooled to room temperature for a few minutes. The SRBC's were centrifuged and resuspended in PBS until obtaining a final concentration of $2 \%$. Equal volumes of both preparations were mixed and poured onto a glass surface with a mold $(18 \times 7.5 \mathrm{~cm})$ and allowed to cool to room temperature. Once the agarose with SRBC's was set, rectangles were cut and placed on slides and kept at $4{ }^{\circ} \mathrm{C}$ in a humidification chamber (maximum 72 hours until use).

Pig small intestine tissue was washed in a $50 \mathrm{mM}$ saline solution and then cut in rectangular sections. The sections were sterilized in a disinfecting solution consisting of 87\% $110 \mathrm{mM}$ saline, 10\% ethanol (PANREAC, ethanol 96\%) and 3\% hydrogen peroxide for 3 hours after which they were left in physiological serum overnight. The next day, the sections were stretched on a glass surface and allowed to dry for a few minutes in the open air. The pretreated sections of the pig's small intestine were placed on the SRBC's agarose rectangles to which a thin layer of anhydrous lanolin for pharmaceutical use was applied.

Based on the results from the TSA assay (section 2.1), one of the following approaches was used for the venom loading experiment:

1. Neutral effect solution: tentacle was incubated on microwells for 5 minutes to carry out the stinging process once it had been incubated.

2. Activator effect solution: solution was applied in spray format on the top of the pig intestine to avoid nematocyst discharge into the microwells during the incubation period.

In order to simulate the sting process in a natural way, the surface of the molds was exposed to approximately $2 \mathrm{~cm}$ of tentacle of $C$. marsupialis, in a linear way, and $3 \mathrm{~cm}$ of tentacle of $P$. noctiluca, in a circular way, during 2 minutes. The circular presentation for $P$. noctiluca was chosen to maximize the venom at one point due to their lower toxicity compared to C. marsupialis.

Afterwards the sting event, the intestine sections were removed and the SRBC's agarose was stored in the humidification chamber at room temperature. After a period of 22 hours, images of the hemolytic areas were obtained with a camera. Hemolytic areas were calculated using Fiji platform [38]. The data was tested by normality and homogenetty by means of the stats package, abailable for the R software platform [39]. As data did not display homogenous variances, a Mann-Whitney test was applied by means 
of the wilcox.test function in order to test if significant differences existed between hemolytic areas. Finally, a graphical representation was performed using the ggplot2 package from the R software platform [40].

Author Contributions: A.B. and V.F. conceived the study; A.B. designed the experiment, participated in the sample collection, performed the laboratory tests, analyzed the data and wrote the manuscript. M.M. participated in the sample collection and contributed to the data analysis. A.S. performed the statistical analyzes and graphical representation. All authors revised the manuscript and contributed to its improvement.

Funding: This research was funded by ISDIN (Barcelona, Spain) and Fundación "la Caixa".

Acknowledgments: The authors gratefully acknowledge the Montgó Research Station (ESCIMO) the collaboration for the collection of Carybdea marsupialis jellyfish, especially to Eva Fontfría and Beatriz Rubio. Thanks to Dr. Angel Yanagihara for the help received during the fine-tuning of the Tentacle skin blood agarose assay methodology. The authors also thank the Medusa team 2016-2018 (Miriam Gentile, Raül Golo, Laura López, Melissa Acebedo and Uxue Tilves) all their knowledge contributed. A.B. was supported by a grant awarded by AGAUR (Agència de Gestió d'Ajuts Universitaris i de Recerca), which allowed presenting the preliminary results of this study in the 6th Jellyfish Bloom Symposium (Cape Town, South Africa). This work acknowledges the 'Severo Ochoa Centre of Excellence' accreditation (CEX2019-000928-S).

Conflicts of Interest: The authors declare no conflict of interest.

Abbreviations: The following abbreviations are used in this manuscript:

$\begin{array}{ll}\text { TSA } & \text { Tentacle Solution Assay } \\ \text { TSBAA } & \text { Tentacle Skin Blood Agarose Assay } \\ \text { PBS } & \text { Phosphate Buffered Saline } \\ \text { SRBC's } & \text { Sheep Red Blood Cells }\end{array}$

\section{References}

1. Daly, M.; Brugler, M.R.; Cartwright, P.; Collins, A.G.; Dawson, M.N.; France, S.C.; McFadden, C.S.; Opresko, D.M.; Rodriguez, E.; Romano, S.; et al. The phylum Cnidaria: A review of phylogenetic patterns and diversity 300 years after Linnaeus. In Linnaeus Tercentenary: Progress in Invertebrate Taxonomy. Zootaxa, 1668; Zhang, Z.-Q.S., Shear, W.A., Eds.; Magnolia Press: Auckland, New Zealand, 2007; pp. 127-182.

2. Zhang, Z. -Q. Animal biodiversity: An update of classification and diversity in 2013. Zootaxa 2013, 3703, 5-11.

3. Mariscal, R.N., 1977. Nematocysts. Coelenterate biology 14, 129-178.

4. Holstein, T.; Tardent, P. An ultrahigh-speed analysis of exocytosis: nematocyst discharge. Science 1984, 223, 830-833. https://doi.org/10.1126/science.6695186

5. Brotz, L.; Cheung, W. W.; Kleisner, K.; Pakhomov, E.; Pauly, D. Increasing jellyfish populations: trends in large marine ecosystems. In Jellyfish Blooms IV; Purcell, J., Mianzan, H., Frost, J.R. Eds.; Sprinter: Dordrecht, 2012, pp. 3-20.

6. Richardson, A. J.; Bakun, A.; Hays, G. C.; Gibbons, M. J. The jellyfish joyride: causes, consequences and management responses to a more gelatinous future. Trends in ecology $\mathcal{E}$ evolution 2009, 24, 312-322. https://doi.org/10.1016/j.tree.2009.01.010

7. Gershwin, L. A.; De Nardi, M.; Winkel, K. D.; Fenner, P. J. Marine stingers: review of an underrecognized global coastal management issue. Coastal Management 2010, 38, 22-41. https://doi.org/10.1080/08920750903345031

8. Graham, W. M.; Gelcich, S.; Robinson, K. L.; Duarte, C. M.; Brotz, L.; Purcell, J. E.; Madin, L.P.; Mianzan, H.; Sutherland, K.R.; Uye, S.; et al. Linking human well-being and jellyfish: ecosystem services, impacts, and societal responses. Frontiers in Ecology and the Environment 2014, 12, 515-523. https://doi.org/10.1890/130298

9. Purcell, J.E.; Uye, S.; Lo, W. Anthropogenic causes of jellyfish blooms and their direct consequences for humans: a review. Mar Ecol Prog Ser 2007, 350, 153-174. https://doi.org/10.3354/meps07093 
10. Remigante, A.; Costa, R.; Morabito, R.; La Spada, G.; Marino, A.; Dossena, S. Impact of scyphozoan venoms on human health and current first aid options for stings. Toxins 2018, 10, 133. https://doi.org/10.3390/toxins10040133

11. Marambio, M.; López, L.; Fuentes, V.L.; Gili, J.M.; Canepa, A. More than 10 years dealing with jellyfish blooms: the jellyfish observation network in the NW Mediterranean. In 6th International Jellyfish Bloom Symposium, Cape Town, South Africa, 2019.

12. Organización Mundial del Turismo (OMT). Panorama del turismo internacional, edición 2019, OMT, Madrid. https://doi.org/10.18111/9789284421237

13. Ariza, E.; Jiménez, J. A.; Sardá, R. A critical assessment of beach management on the Catalan coast. Ocean \& Coastal Management 2008, 51, 141-160. https://doi.org/10.1016/j.ocecoaman.2007.02.009 14. Boulware, D. R. A randomized, controlled field trial for the prevention of jellyfish stings with a topical sting inhibitor. Journal of travel medicine 2006, 13, 166-171. https://doi.org/10.1111/j.17088305.2006.00036.x

15. Bordehore, C.; Alonso, C.; Sánchez-Fernández, L.; Canepa, A.; Acevedo, M.; Nogué, S.; Fuentes, V.L. Lifeguard assistance at Spanish Mediterranean beaches: Jellyfish prevail and proposals for improving risk management. Ocean \& coastal management 2016, 131, 45-52. https://doi.org/10.1016/j.ocecoaman.2016.08.008

16. Marambio, M. (ICM-CSIC - Institute of Marine Sciences, Barcelona, Spain). Personal comunication, 2020.

17. Guía de identificación y tratamiento de picaduras de medusas y otros organismos gelatinosos de la costa mediterránea española. Available online: http://icmdivulga.icm.csic.es/identifica-lasmedusas-del-mediterraneo/ (accessed on 25 March 2020).

18. Mariottini, G.L.; Giacco, E., Pane, L. The Mauve Stinger Pelagia noctiluca (Forsskål, 1775). Distribution, ecology, toxicity and epidemiology of stings. A review. Mar. Drugs 2008, 6, 496-513. https://doi.org/10.3390/md20080025

19. Mariottini, G.L.; Pane, L. Mediterranean jellyfish venoms: A review on scyphomedusae. Mar. Drugs 2010, 8, 1122-1152. https://doi.org/10.3390/md8041122

20. Mathieu, L.; Blomet, J.; Hall, A. Pelagia Noctiluca Jellyfish: Can Lesions and Symptoms be Prevented or Ameliorated?. J Marine Biol Aquacult 2018, 4, 48-52. https://doi.org/10.15436/23810750.18.1971

21. Montgomery, L.; Seys, J.; Mees, J. To pee, or not to pee: a review on envenomation and treatment in European jellyfish species. Mar. Drugs 2016, 14, 127. https://doi.org/10.3390/md14070127

22. Bordehore, C.; Fuentes, V.; Atienza, D.; Barberá, C.; Fernandez-Jover, D., Roig, M.; AcevedoDudley, M.J.; Canepa, A.J.; Gili, J. Detection of an unusual presence of the cubozoan Carybdea marsupialis at shallow beaches located near Denia, Spain (south-western Mediterranean). Marine Biodiversity Records 2011, 4, E69. https://doi.org/10.1017/S1755267211000650

23. Milla, L.; Segura-Puertas, L.; Celis, L.; Heimer de la Cotera, E. Dermatitis por contacto con Carybdea marsupialis (cnidaria cubozoa). Dermatología Revista Mexicana 2000, 44, 167-170.

24. Peca, G.; Rafanelli, S.; Galassi, G.; Bartolo, P. D.; Bertini, S.; Alberani, M., Beccari, G. Contact reactions to the jellyfish Carybdea marsupialis: observation of 40 cases. Contact dermatitis 1997, 36, 124126. https://doi.org/10.1111/j.1600-0536.1997.tb00392.x

25. Killi, N.; Mariottini, G.L. Cnidarian Jellyfish: Ecological aspects, nematocyst isolation, and treatment methods of Sting. In Marine Organisms as Model Systems in Biology and Medicine; Kloc, M., Kubiak, J. Eds.; Springer: Cham, 2018, pp. 477-513.

26. Cegolon, L.; Heymann, W.C.; Lange, J.H.; Mastrangelo, G. Jellyfish stings and their management: a review. Mar. drugs 2013, 11, 523-550. https://doi.org/ 10.3390/md11020523

27. Doyle, T. K.; Headlam, J.L.; Wilcox, C.L.; MacLoughlin, E.; Yanagihara, A.A. Evaluation of Cyanea capillata sting management protocols using ex vivo and in vitro envenomation models. Toxins 2017, 9, 215. https://doi.org/10.3390/toxins9070215

28. Birsa, L.M.; Verity, P.G.; Lee, R.F. Evaluation of the effects of various chemicals on discharge of and pain caused by jellyfish nematocysts. Comparative Biochemistry and Physiology Part C: Toxicology $\mathcal{E}$ Pharmacology 2010, 151, 426-430. https://doi.org/10.1016/j.cbpc.2010.01.007

29. Pyo, M. J.; Lee, H.; Bae, S. K.; Heo, Y.; Choudhary, I.; Yoon, W. D.; Kang. C.; Kim, E. Modulation of jellyfish nematocyst discharges and management of human skin stings in Nemopilema nomurai and Carybdea mora. Toxicon 2016, 109, 26-32. https://doi.org/10.1016/j.toxicon.2015.10.019

30. Hartwick, R.; Callanan, V.; Williamson, J. Disarming the box jellyfish: Nematocyst inhibition in Chironex fleckeri. Med. J. Aust. 1980, 1, 5-20. https://doi.org/10.5694/j.1326-5377.1980.tb134566.x

31. Fenner, P.J.; Williamson, J. Experiments with the nematocysts of Carybdea rastoni ("Jimble"). Med. J. Aust. 1987, 147, 258-259. https://doi.org/10.5694/j.1326-5377.1987.tb133433.x 
32. Yanagihara, A.A.; Wilcox, C.; King, R.; Hurwitz, K.; Castelfranco, A.M. Experimental assays to assess the efficacy of vinegar and other topical first-aid approaches on cubozoan (Alatina alata) tentacle firing and venom toxicity. Toxins 2016, 8, 19. https://doi.org/10.3390/toxins8010019

33. Yanagihara, A.A.; Wilcox, C.L. Cubozoan sting-site seawater rinse, scraping, and ice can increase venom load: upending current first aid recommendations. Toxins 2017, 9, 105. https://doi.org/10.3390/toxins9030105

34. Fenner, P.J.; Fitzpatrick, P.F. Experiments with the nematocysts of Cyanea capillata. Med. J. Aust. 1986, 145, 174.

35. Morabito, R.; Marino, A.; Dossena, S.; La Spada, G. Nematocyst discharge in Pelagia noctiluca (Cnidaria, Scyphozoa) oral arms can be affected by lidocaine, ethanol, ammonia and acetic acid. Toxicon 2014, 83, 52-58. https://doi.org/10.1016/j.toxicon.2014.03.002

36. Salleo, A.; La Spada, G.; Falzea, G.; Denaro, M. G. Discharging effect of anions and inhibitory effect of divalent cations on isolated nematocysts of Pelagia noctiluca. Molecular physiology 1984, 5, 2533.

37. Colin, S.P.; Costello, J.H. Functional characteristics of nematocysts found on the scyphomedusa Cyanea capillata. Journal of Experimental Marine Biology and Ecology 2007, 351, 114-120. https://doi.org/10.1016/j.jembe.2007.06.033

38. Schindelin, J.; Arganda-Carreras, I.; Frise, E.; Kaynig, V.; Longair, M.; Pietzsch, T.; Preibisch, S.; Rueden, C; Saalfeld, S.; Schmid , B; et al. Fiji: an open-source platform for biological-image analysis. Nature methods 2012, 9, 676-682.

39. R: A language and environment for statistical computing. R Foundation for Statistical Computing. Available online: https://www.R-project.org/ (accessed on 08 March 2020).

40. Wickham, H. ggplot2: Elegant Graphics for Data Analysis. Springer-Verlag: New York, 2016. ISBN 978-3-319-24277-4. https://ggplot2.tidyverse.org. 